\title{
Kinematic Quality of Reaching Movements in Preterm Infants
}

\author{
BJØRG FALLANG, OLA DIDRIK SAUGSTAD, JENS GRØGAARD, AND \\ MIJNA HADDERS-ALGRA \\ Oslo University College [B.F.], Health Sciences, Physiotherapy Programme, 0167 Oslo, Norway; \\ Department of Pediatric Research [O.D.S.], The National Hospital, University of Oslo, 0027 Oslo, \\ Norway; Ullevaal University Hospital [J.G.], Child Centre, N-0407 Oslo, Norway; and Department of \\ Neurology [M.H.-A.], Developmental Neurology, University of Groningen, Hanzeplein 1, 9713 GZ \\ Groningen, The Netherlands
}

\begin{abstract}
ABST
Many preterm infants may experience so-called minor devel-
opmental disorders; however, in general, the problems in motor
behavior are not detected until school age. To introduce therapies
aimed at the prevention of these problems, we need to increase
our knowledge of motor function and dysfunction at early age.
The present study focused on the organization of reaching move-
ments in full-term and preterm infants without cerebral palsy.
The reaching behavior of premature infants ( $n=63$ ) was as-
sessed longitudinally at the corrected ages of 4 and 6 mo. Clinical
assessments were made at 6 and 12 mo of age. On the basis of the
infant's morbidity during the early stay in the neonatal intensive
care unit, the preterm infants were allocated into a high-risk and
a low-risk group. Results from a previous study in full-term
infants $(n=13)$ were included. Kinematics of reaching move-
ments in supine position were measured, and the analysis focused
on movement velocity and movement units. A compound param-
eter of kinematic variables was created, reflecting the quality of
reaching movements. The present study showed that at the age of
4 mo, low-risk preterm infants showed more often optimal
\end{abstract}
There is an increasing awareness that many preterm infants may experience subtle long-term morbidities. Usually these so-called minor developmental disorders, such as clumsiness and specific learning disorders, are identified first at school age (1-3). It has been questioned whether these minor developmental disorders can be detected at early age, so that intervention aiming at the prevention of problems that interfere with academic achievement could be introduced timely.

Recently, evidence has been provided that early motor development in preterm infants without major developmental disorders differs from that of full-term (FT) infants. The ma-

Received January 7, 2002; accepted November 21, 2002.

Correspondence: Bjørg Fallang, Oslo University College, Health Sciences, Physiotherapy Programme, Pilestredet 52, 0167 Oslo, Norway; e-mail: bjorg.fallang@hf.hio.no

This study was supported by The Norwegian Fund for Postgraduate Training in Physiotherapy, Norway.

DOI: 10.1203/01.PDR.0000058925.94994.BC reaching behavior than full-term and preterm high-risk infants. This better reaching performance was related to a better general motor and behavioral development during the first year of life. At the age of $6 \mathrm{mo}$, the advantage of the low-risk group in reaching behavior had disappeared and a disadvantage in the form of nonoptimal reaching behavior of the high-risk group emerged. (Pediatr Res 53: 836-842, 2003)

FT, full-term
HR, high-risk
IBR, Infant Behavior Record (of Bayley test)
LR, low-risk
MDI, Mental Developmental Index
MU, movement units
PDI, Psychomotor Developmental Index
PT, preterm
ROP, retinopathy of prematurity

jority of studies focused on postural development. They indicated that so-called "low-risk" preterm infants show significantly more often an exaggerated trunk extension $(4,5)$ or "transient dystonia" $(6,7)$ than FT infants. Van der Fits et al. (8), who studied the development of postural adjustments during reaching, reported that low-risk (LR) preterm infants showed an excessive amount of postural activity. Moreover, the postural adjustments of the preterm infants were temporally disorganized and could not be modulated to task-specific conditions, such as the velocity of the reaching arm and sitting position. The mild dysfunctions in postural control of preterm infants are related to a poorer quality of reaching and grasping at the age of $1 \mathrm{y}(9,10)$ and possibly also to motor dysfunctions (7) or learning problems (6) at school age.

Little is known on the early development of reaching and grasping in preterm infants - despite that the emergence of goal-directed motility in the arms forms the first phase of the 
development of voluntary motor control during infancy. Clinical studies indicated that the quality of reaching and grasping behavior in LR preterm infants is less satisfactory than that in FT infants $(9,11,12)$. To the best of our knowledge no data have been reported on the development of kinematic characteristics of reaching movements in preterm infants. Studies on the kinematics of reaching in FT infants have resulted in substantial insight into the development of motor control during reaching (13-16). They showed that reaching movements become more straight-lined and exhibit less corrections of the movement path with increasing age. These corrections are termed movement units (MU). MU are submovements of the reaching movement, which are determined with the help of peaks in the velocity profile of the reaching hand $(13,14)$. Normal development is characterized not only by a decrease of the number of MU but also by an increase of the relative size of the first MU, i.e. the so-called transport unit. During the first half year of life, the kinematic characteristics of reaching depend on the child's position: reaching movements in supine consist of more MU than those performed in a secured sitting position $(17,18)$.

The aim of the present study was to improve our insight into the specific dysfunctions of preterm infants during the development of reaching. To this end, kinematic parameters of goal-directed reaching in supine were recorded at the ages of 4 and 6 mo corrected age in two groups of preterm infants without cerebral palsy. One group consisted of infants with various neonatal complications [high-risk (HR) group] and the other of preterm infants without neonatal problems (LR group). The data were compared with those of FT infants (17). Possible correlations between the characteristics of reaching and neonatal morbidity and general development at 6 and 12 mo were evaluated. We addressed the following questions: 1) Do reaching movements of preterm infants without cerebral palsy differ in kinematic characteristics from those of FT infants at the corrected ages of 4 and 6 mo? If they do, then 2) can the differences be explained by the presence of "high risk," gestational age at birth, a birth weight below the 10th percentile, respiratory illness, or mild abnormalities on the neonatal ultrasound scans of the brain? 3) Are the characteristics of reaching in preterm infants related to motor and mental development at the corrected ages of 6 and $12 \mathrm{mo}$ ?

\section{METHODS}

Subjects. The premature infants were recruited from three hospitals that cover the city of Oslo and the nearest county at $36 \mathrm{wk}$ postmenstrual age. The primary inclusion criteria were a birth weight $<1750 \mathrm{~g}$ and a mother speaking either a Scandinavian language or English. Infants with serious illness at $36 \mathrm{wk}$ or in whom a high risk for the development of cerebral palsy was diagnosed, i.e. infant with cystic periventricular leukomalacia or intracranial hemorrhages extending into the parenchyma, were excluded. This resulted in a group of 88 infants. After inclusion, another two parents denied further participation, and families of four infants moved out of the area. Eight infants were excluded at a later stage because of serious illness or cerebral palsy, leaving the study with 74 infants. The absence of cerebral palsy in the infants included in the study was confirmed at the clinical examination at 1 y (19) and in a recent follow-up at the age of $5 \mathrm{y}$, which included a detailed neurologic assessment according to Touwen (20). The group of 74 preterm infants was divided into an HR and a LR group on the basis of the following criteria. High risk was assigned in case the infant had experienced one or more of the following risk factors: 1) Apgar score at $5 \mathrm{~min}<3,2$ ) at least one of the following respiratory problems: $a$ ) respiratory illness needing surfactant treatment, $b)>1$ wk of mechanical ventilation, c) pneumothorax, or $d$ ) persistent ductus arteriosus (21, 22). The diagnosis persistent patent ductus arteriosus was based on clinical and echocardiographic data (23). Four infants had retinopathy of prematurity (ROP), one a grade 1 , two a grade 2, and the fourth a grade 3 . None of the infants had ROP plus disease (24).

We aimed at recording reaching movements of all infants at the corrected ages of 4 and 6 mo. At the age of 4 mo, successful kinematic recordings were obtained in 44 infants. No kinematic data were available in the rest because of absence of reaching behavior $(n=10)$, technical problems $(n=13)$, minor illnesses $(n=3)$, or family holidays $(n=4)$. At 6 mo, we achieved 41 successful recordings. Kinematic data were missing because of absence of reaching behavior $(n=1)$, technical problems $(n=19)$, minor illnesses of the child $(n=$ 6 ), or family holidays $(n=7)$. The neonatal characteristics of the 30 respectively 33 infants in whom no reaching data could be recorded did not differ from those of the 44 respectively 41 preterms in whom the kinematics of reaching could be obtained at 4 and 6 mo. The clinical data on the two preterm groups assessed at 4 and 6 mo are summarized in Table 1.

Thirteen healthy infants who were born at FT and who participated in a similar project on the development of reaching served as control infants (17). These infants who were recruited at Ullevaal Hospital, Oslo, were born at a mean gestational age of 40 wk and $6 \mathrm{~d}$ with a mean birth weight of $3878 \mathrm{~g}$ (SD \pm 442 ). The Ethics Committee of region 1 of Norway approved the study, and all parents signed informed consent before participation.

Procedure. During the reaching assessment, the infant was placed in supine position on the floor with a toy attached to a horizontal rod, suspended approximately $12 \mathrm{~cm}$ above the infant's chest. Reflective markers were attached to the infant's hands and the toy. The experimenter was kneeling behind the infant's head and gently held the infant's forearms toward the floor and released the arms, when the infant was calm and not pushing the arms toward the experimenter's hands. The recording started just before the arm was released and lasted for $15 \mathrm{~s}$. Three reaching movements (contact with the toy) per infant were recorded and stored on a computer and on videotapes for off-line analysis. Only reaches during which the infants looked at the toy and touched it were defined as goal-directed and successful. Care was taken that the infant was in an alert, noncrying behavioral state. Table 2 shows the number of trials analyzed.

The kinematics of the reaching movements were recorded with either the MacReflex movement analysis system (Qualisys, Gothenburg, Sweden; $n=52$ infants) or the Peak Perfor- 
Table 1. Clinical details of the groups of preterm infants

\begin{tabular}{|c|c|c|c|c|}
\hline & \multicolumn{2}{|c|}{ HR group } & \multicolumn{2}{|c|}{ LR group } \\
\hline & 4 mo $(n=16)$ & $6 \mathrm{mo}(n=15)$ & 4 mo $(n=28)$ & $6 \mathrm{mo}(n=26)$ \\
\hline Sex: M/F & $7 / 9$ & $9 / 6$ & $17 / 11$ & $12 / 14$ \\
\hline Gestational age at birth [median (range)] & $27(24-33)$ & $27(24-33)$ & $30(26-34)$ & $31(26-34)$ \\
\hline SGA $[n(\%)]$ & $4(25)$ & $5(33)$ & $13(46)$ & $13(50)$ \\
\hline Mechanical ventilation $>1$ wk $(n)$ & 10 & 8 & 0 & 0 \\
\hline Normal US data & 5 & 7 & 10 & 11 \\
\hline IVH & 0 & 0 & 1 & 1 \\
\hline PVE & 3 & 2 & 0 & 0 \\
\hline $\mathrm{PVE}+\mathrm{IVH}$ & 0 & 0 & 1 & 0 \\
\hline Ventricles $\uparrow$ & 0 & 0 & 0 & 1 \\
\hline Ventricles $\uparrow+\mathrm{IVH}$ & 0 & 0 & 1 & 1 \\
\hline
\end{tabular}

SGA, small for gestational age, birth weight $\leq 10 \%$ (37); PDA, persistent ductus arteriosus; US, ultrasound; IVH, intraventricular haemmorhage; PVE, periventricular echodensities, i.e. echodensities present for at least $14 \mathrm{~d}$.

Table 2. Summary of number of subjects and reaching trials included in the study

\begin{tabular}{|c|c|c|c|c|c|c|}
\hline \multirow[b]{2}{*}{ Group } & \multicolumn{2}{|c|}{$\begin{array}{l}\text { Adequate reaching data at } 4 \text { and } 6 \\
\text { mo }\end{array}$} & \multicolumn{2}{|c|}{ Adequate reaching data at 4 mo only } & \multicolumn{2}{|c|}{ Adequate reaching data at 6 mo only } \\
\hline & Subjects $(n)$ & $\begin{array}{l}\text { Total number of } \\
\text { trials analyzed }\end{array}$ & Subjects $(n)$ & $\begin{array}{l}\text { Total number of } \\
\text { trials analyzed }\end{array}$ & Subjects $(n)$ & $\begin{array}{l}\text { Total number of } \\
\text { trials analyzed }\end{array}$ \\
\hline PT-LR & 19 & 38 and 47 & 9 & 24 & 7 & 20 \\
\hline PT-HR & 9 & 27 and 13 & 7 & 17 & 6 & 14 \\
\hline FT & 8 & 22 and 22 & 1 & 1 & 3 & 10 \\
\hline
\end{tabular}

PT, preterm.

mance Movement Analysis System (Peak Performance Technologies, Inglewood, CO, U.S.A.; $n=7$ infants). The data processing in both systems was identical. The kinematic systems recorded the reaching movements of the hand in threedimensional space by using four cameras (see 17). For the purpose of visual identification of markers, the whole session was videotaped from a lateral view.

Neonatal morbidity of the children was collected in a standardized way. In $50 \%$ to $67 \%$ of the infants, data on serial neonatal ultrasound scans of the brain were available. The scans were made with a $7.5-\mathrm{MHz}$ transducer. Periventricular hemorrhages and echodensities were classified according to the methods of Levene et al. (25) and Jongmans et al. (26) (see Table 1).

The infant's level of motor and mental development was quantified with the help of the Bayley Scales of Infant Development Psychomotor Developmental Index (PDI) and Mental Developmental Index (MDI) (19), respectively, at the corrected ages of 6 and 12 mo. During the Bayley assessments, the infant's behavior was scored the by means of the Infant Behavior Record (IBR).

Data analysis. The data from both kinematic systems were exported for analysis to the same custom-made program (M. Guttormsen, Norwegian University of Sports and Physical Education, Oslo, Norway). The data were filtered with a zerophased forward/backward second-order Butterworth filter with cutoff frequency of $6 \mathrm{~Hz}$. Reaching onset was defined as the video frame when the hand started to move from a quiet resting position toward the toy. When the hands were in contact with the toy, the reach ended. The contact with the toy was indicated by an increase in the velocity profile of the toy. The MU were distinguished with the help of the hand velocity peak profile $(13,14)$. The MU were defined from the velocity profile by three characteristics: 1) at the beginning of the unit, the velocity had to increase by $20 \mathrm{~mm} / \mathrm{s} ; 2$ ) the velocity peak of the MU had to be $>10 \%$ of the $V_{\max }$ for the whole trial; 3) the local maximum of a velocity peak was defined as the highest peak between three frames to each side. The analysis of the hand movements focused on 1) the number of MU, 2) the length of the first MU relative to total movement path, 3) the duration of the first MU relative to total movement time, 4) maximal hand velocity during each reach, and 5) total movement time. For details see (17).

Statistics. In the analysis of the reaching data, individual median values were calculated for each infant at each age. For evaluating the differences in kinematics among the three groups the nonparametric Mann-Whitney test was used. The nonpaired Mann-Whitney was also used to assess the effect of age on kinematic development. Mann-Whitney was used instead of Wilcoxon because of missing data at both ages. When kinematic data were dichotomized, $\chi^{2}$ or Fisher exact test was performed. 
The differences in developmental outcome among the three groups were assessed with ANOVA. The relations between reaching behavior and neonatal data and developmental outcome were assessed with $\chi^{2}$ or Fisher exact test (e.g. difference in presence of optimal reaching behavior between infants with and without abnormalities on the ultrasound scans of the brain), Spearman correlation (e.g. correlation between gestational age at birth and number of MU), and Mann-Whitney (e.g. difference in Bayley MDI between infants with and without optimal reaching behavior). Statistical calculations were performed with SPSS version 8. Differences with $p<$ 0.05 were considered to be statistically significant (two tailed).

\section{RESULTS}

Motor and mental development. The HR group scored worse than the FT group on the Bayley PDI at 6 mo (mean scores 83 versus 97 , ANOVA, $\mathrm{F}_{2,64}=3.87, p=0.03$ ), but this difference disappeared at $12 \mathrm{mo}$. The Bayley PDI scores at 6 and $12 \mathrm{mo}$ of the LR group did not differ significantly from those of the FT group. The HR infants also performed worse than the FT infants on the Bayley MDI at 6 mo (mean scores 96 versus 106; ANOVA, $\left.\mathrm{F}_{2,63}=6.75, p<0.002\right)$, but this difference did persist until 12 mo (mean scores 91 versus 107; ANOVA, $\left.\mathrm{F}_{2,64}=8.99, p<0.001\right)$. At $12 \mathrm{mo}$, the HR group also scored worse on the MDI than the LR group (mean scores 91 versus 101; ANOVA, $\left.\mathrm{F}_{2,64}=6.75, p<0.004\right)$. The MDI scores of the LR group did not differ significantly from those of the FT group. Two HR infants had at 12 mo an MDI $<70$.

The total behavioral rating on the IBR of the HR infants at 6 mo was significantly lower than that of the FT infants (mean scores 117 versus 125; ANOVA, $\mathrm{F}_{2,60}=4.81, p<0.02$ ). The HR group scored also significantly worse on the subscale orientation/engagement of the IBR than the LR group; the difference with the FT group failed to reach significance (mean scores of the three groups: 44, 47, and 47 respectively; ANOVA, $\left.\mathrm{F}_{2,62}=5.46, p<0.007\right)$. Behavioral scores of the LR and FT groups did not differ. At the age of $12 \mathrm{mo}$, preterm and FT infants did not differ in the total IBR scores, but a remarkable difference was observed in the orientation/ engagement subscale with the preterm infants scoring higher than the FT infants (mean scores FT, 40; LR, 48; HR, 45; ANOVA, $\mathrm{F}_{2,32}=10.68, p<0.001, p=0.03$, respectively).

Kinematics of reaching behavior. The portion of infants who did not reach successfully at 4 mo did not differ significantly among the three groups. Five $(13 \%)$ of the 38 LR infants, five (20\%) of the $25 \mathrm{HR}$ infants, and three (25\%) of the 12 FT infants failed to reach the toy.

At 4 mo of age, the kinematic characteristics of the LR infants differed significantly from those of the FT infants (Table 3). The reaching movements of the LR preterm infants had less movement units (Mann-Whitney, $p=0.01$ ) and took less time than those of the FT infants $(p=0.02)$. In addition, in the LR preterm infants, the first MU, i.e., the transport unit, represented a larger part of the reaching movement $(p=0.01)$ than it did in the FT infants. Between the LR and HR preterm groups, no significant kinematic differences were present at 4 mo. At the age of $6 \mathrm{mo}$, the kinematics of reaching did not differ among the three groups (Table 3). Developmental changes from 4 to $6 \mathrm{mo}$ in reaching characteristics were significant in the FT group (Table 3). Similar changes were present to a minor extent in the HR preterm infants and absent in the LR preterm infants.

The finding that the preterm infants performed better than the FT infants at 4 mo was puzzling. It did not fit with our Gestalt impression of reaching behavior observed on the video recordings, where especially velocity aspects of the movement caught our attention. Apparently, the single parameters such as MU or movement time did not catch the observable qualitative differences in movement performance between the preterm and FT infants. Therefore, we decided to create a compound variable, "kinematic movement quality," consisting of a combination of the variables peak velocity and number of MU. Movement velocity and the number of MU were both divided into three groups: low $(<301 \mathrm{~m} / \mathrm{s})$, medium $(301-800 \mathrm{~m} / \mathrm{s})$, and high $(>800 \mathrm{~m} / \mathrm{s})$ velocity, and a low $(<3.5)$, medium (3.5-6), and high $(>6)$ number of MU. The various combinations of movement velocity and number of MU resulted in nine $(3 \times 3)$ possible scores of kinematic movement quality. The type of kinematic movement quality that the infant exhibited most frequently during the three reaching movements determined the infant's class of kinematic movement quality. In case two forms of quality were equally often present, the infant was given the best quality of the two.

Figure 1 shows the distribution of the preferred kinematic movement quality in the three groups at 4 and $6 \mathrm{mo}$. It indicates that the quality of reaching behavior in preterm infants is more variable than that of FT infants. The majority of 6-mo-old FT infants showed reaching movements with a medium velocity and a few MU, a finding that is in agreement with literature data $(14,17)$. We classified this movement

Table 3. Kinematic characteristics of reaching in preterm and FT infants

\begin{tabular}{|c|c|c|c|c|c|c|}
\hline \multirow[b]{2}{*}{ Kinematic parameters } & \multicolumn{3}{|c|}{$4 \mathrm{mo}$} & \multicolumn{3}{|c|}{$6 \mathrm{mo}$} \\
\hline & $\begin{array}{c}\text { PT-HR } \\
(n=16)\end{array}$ & $\begin{array}{c}\text { PT-LR } \\
(n=28)\end{array}$ & $\begin{array}{c}\text { FT } \\
(n=9)\end{array}$ & $\begin{array}{l}\text { PT-HR } \\
(n=15)\end{array}$ & $\begin{array}{c}\text { PT-LR } \\
(n=26)\end{array}$ & $\begin{array}{c}\text { FT } \\
(n=11)\end{array}$ \\
\hline No. of MU & 5.5 & $3.8 * *$ & 7.0 & $4.0 \dagger$ & 2.0 & $3.0 \dagger \dagger$ \\
\hline Peak velocity $(\mathrm{m} / \mathrm{s})$ & 600 & 496 & 631 & 390 & 564 & 550 \\
\hline Travel length of first MU & $0.29 *$ & $0.4 * *$ & 0.12 & 0.40 & 0.51 & 0.39 \\
\hline Duration of first MU & 0.22 & 0.32 & 0.21 & 0.38 & 0.43 & $0.29 \dagger$ \\
\hline Total time (s) & 1.52 & $1.08^{*}$ & 1.64 & 0.94 & 0.73 & $1.24 \dagger$ \\
\hline
\end{tabular}

Differences between preterm and FT infants (Mann-Whitney): $* p<0.05, * * p<0.01$.

Differences between 4 and 6 mo (Mann-Whitney): $\uparrow p<0.05$, $\dagger p p<0.01$. 


\section{Preference patterns}
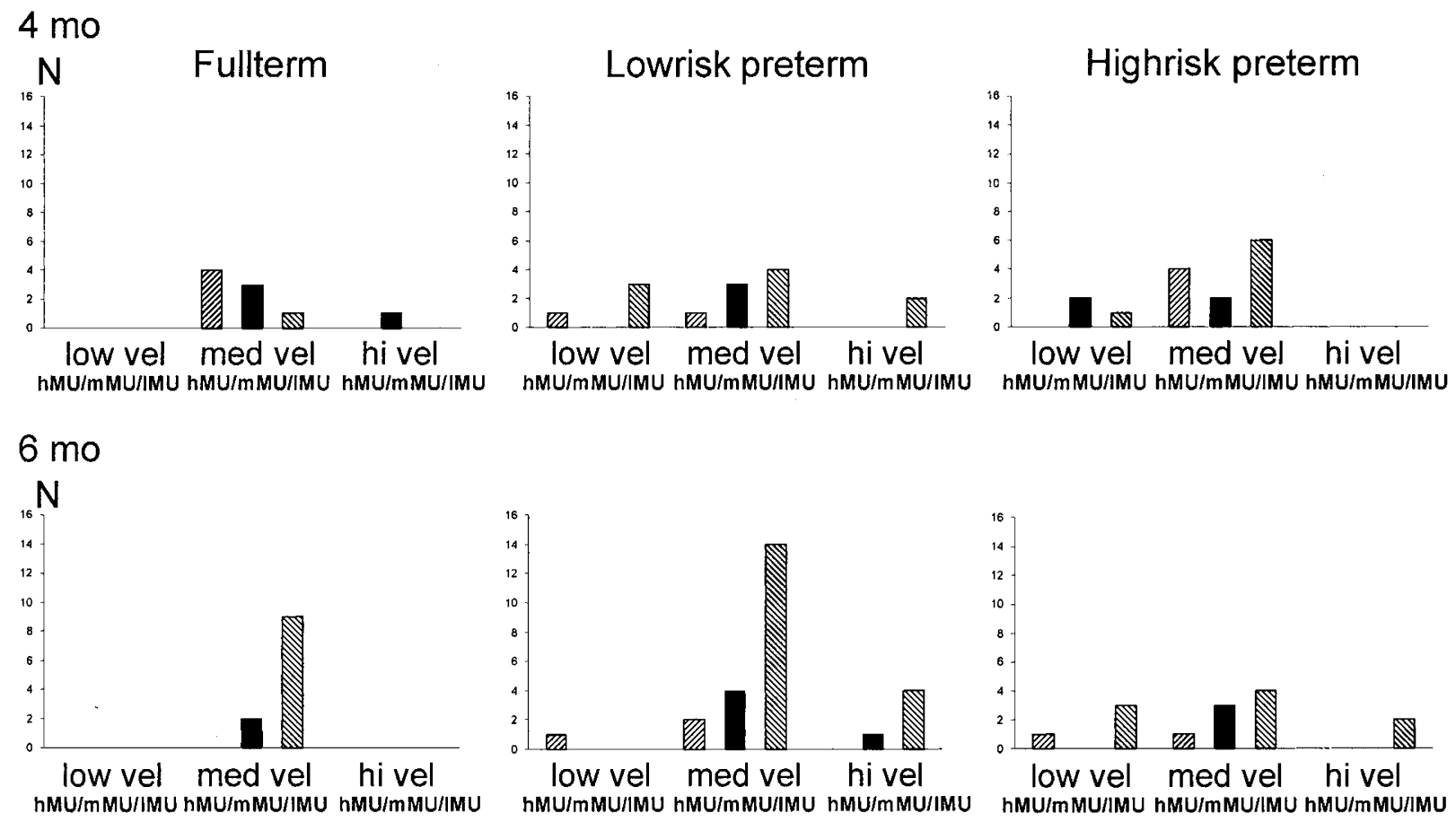

Figure 1. Distribution of the infants' most frequently used kinematic movement quality in the three study groups at 4 and 6 mo of age. VEL, velocity, divided into low, medium (med), and high (hi). MU divided into high (h), medium (m), and low (l) number.

quality as optimal. At the age of 4 mo, only one (11\%) of the nine FT infants showed this optimal movement quality. In the group of LR infants, $15(53 \%)$ of 28 already exhibited this movement quality at 4 mo (difference with FT group: Fisher $p$ $=0.05)$. In the HR group, six (40\%) of 15 infants showed an optimal reaching behavior, a frequency that did not differ from the other two groups. At 6 mo, nine (82\%) of 11 FT infants, 14 $(68 \%)$ of 26 LR infants, and four (29\%) of 14 HR infants had optimal reaching movements. This means that the HR infants showed significantly less often optimal movements than both FT and LR infants (Fisher $p=0.02, p=0.05$, respectively). It was remarkable that four of the nonoptimally moving HR infants showed reaching movements with a low velocity, as movements with a low velocity occurred in none of the FT and one of the LR infants (Fig. 1).

Clinical data and reaching characteristics in preterm infants. In the preterm infants, no relation was found between neonatal characteristics such as birth weight, gestational age at birth, and respiratory morbidity and the kinematics of reaching at 4 and $6 \mathrm{mo}$. Neither were minor abnormalities present on the neonatal ultrasound scans of the brain correlated to reaching behavior at 4 and $6 \mathrm{mo}$. Weight and length of the infants at 4 and 6 mo did not influence the kinematics of reaching. Reaching behavior of the four infants with ROP did not differ from those without ROP.

In the FT group, the kinematics of reaching were not related to the Bayley MDI and PDI at 6 and $12 \mathrm{mo}$. In the LR infants at 4 mo, optimal reaching (reaching with medium velocity and few MU) was related to a better performance on the Bayley
PDI at 6 and 12 mo (Mann-Whitney, $p=0.001, p<0.005$, respectively). At $6 \mathrm{mo}$, reaching behavior in both the LR and the HR infants was not related to Bayley MDI or PDI scores at 6 and 12 mo. The relationships between reaching characteristics and Bayley scores did not change when the two infants with an MDI $<70$ at 12 mo were excluded from the analysis.

In the FT group, the kinematics of reaching at 4 mo was not related to behavioral scoring (IBR) at 6 and 12 mo. However, in FT infants at $6 \mathrm{mo}$, a shorter total reaching time was related to a better total IBR score at $12 \mathrm{mo}(r=0.83, p=0.04)$. In the LR infants at $4 \mathrm{mo}$, less MU, a shorter total reaching time, and a longer relative duration and displacement of the first MU were related to a better IBR score at $12 \mathrm{mo}(r=0.49-0.56, p$ $<0.04$ ). In addition, less MU and a larger relative displacement of the first MU were related to a better orienting behavior at $12 \mathrm{mo}(r=0.47-0.49, p<0.05)$ and optimal reaching movements to better orientation and a higher total IBR score at 12 mo (Mann-Whitney, $p=0.001, p=0.003$ ). In the LR group, the kinematics of reaching at 6 mo were not related to behavior at 6 and $12 \mathrm{mo}$. In the HR group, no relationships were found between reaching data and behavioral development. Social class defined as the mother having below or above $12 \mathrm{y}$ of education did not influence kinematic results and Bayley mental or behavioral scores.

\section{DISCUSSION}

The present study showed that at the age of 4 mo, LR preterm infants show more often optimal reaching behavior 
than FT and preterm HR infants, despite that LR infants more often had a birth weight below the 10th percentile than the HR infants (Table 1). This better reaching performance was related to a better general motor and behavioral development during the first year of life. At the age of $6 \mathrm{mo}$, the advantage of the LR group in reaching behavior had disappeared and a disadvantage in the form of nonoptimal reaching behavior of the HR group emerged.

The finding of a better performance in reaching behavior of the LR infants at the age of 4 mo could be interpreted as a sign of advanced and positive development. This notion is supported by the observation that optimal reaching was related to better general developmental outcome at the age of $1 \mathrm{y}$. It might suggest that LR preterm infants can take advantage of extra early extrauterine experience in the development of reaching. The issue of whether the additional extrauterine experience of preterm infants is beneficial or deleterious for developmental progress is a matter of debate (27). The majority of studies assessing development of preterm infants focus on dysfunctions and disorders. Few studies address heterogeneity in outcome of preterms without serious morbidity. Studies that deal with various types of development are found in particular in the field of the development of vision. Evidence has been provided that LR preterm infants show an accelerated maturation of visual evoked responses in the neonatal period (28) and of visual fields during the first weeks postterm (29). At older ages, the differences in visual development between LR preterm infants and FT infants disappear (28). Eilers et al. (30), who addressed differences in speech development, found that preterm infants developed canonical babbling at an earlier age than FT infants did. It is conceivable that the advantages that preterm infants might have from early extrauterine experience are subtle, mostly transient, and often occluded by the negative effects associated with preterm birth. Possibly a beneficial effect of preterm birth can be found only in LR preterm infants and only when the study is carried out in sufficient detail.

Reaching performance of the group of HR infants started to differ from that of the FT infants at the age of $6 \mathrm{mo}$. At that age, the HR infants showed more often nonoptimal movements that consisted of movements with a too high or too low velocity and/or movements with too many MU. This might suggest that the infants with nonoptimal movements have a dysfunction in the capacity to modulate and fine-tune motor output during reaching. A similar type of dysfunctional fine-tuning has been reported to occur in preterm infants during the first $18 \mathrm{mo}$ of life in postural muscle activity during reaching (8). Possibly a dysfunctional modulation of muscle activity is also the mechanism explaining the frequently reported postural dysfunctions of preterm infants, such as transient dystonia $(4,5,7)$. Remarkably, the nonoptimal reaching of the HR infants was not related to developmental outcome at the age of $12 \mathrm{mo}$. The absence of such a relation might be explained by the short duration of follow-up, as it usually takes developmental time before minor dysfunctions that interfere with school performance emerge (31). Interestingly, we found that reaching behavior at $4 \mathrm{mo}$ was more strongly related to developmental outcome than reaching behavior at $6 \mathrm{mo}$. Others have indicated that around 6 mo, a transition in motor development occurs $(32,33)$. Possibly, this phase of transition blurs relationships between early and later signs of neural dysfunction.

Reaching behavior at 4 and 6 mo was not related to neonatal morbidity, including mild abnormalities present on the neonatal ultrasound scans of the brain. With respect to the latter, it should be kept in mind that ultrasound data were not available for all infants. Nevertheless, a similar absence of relationships between ultrasound findings and minor developmental disorders has been reported before $(8,34)$. It could be surmised that the nonoptimal motor behavior of some preterm infants is an expression of mild abnormalities of the brain, which escape the ultrasonographer's eye but which might be detected with the help of other techniques such as magnetic resonance imaging, in particular diffusion-weighted imaging $(35,36)$.

\section{CONCLUSION}

In conclusion, LR preterm infants may show a temporary acceleration of the development of reaching - which is related to a better functional outcome at the age of $12 \mathrm{mo}$. HR preterm infants seem to grow into dysfunctional reaching behavior at the age of $6 \mathrm{mo}$. The significance of the latter finding should be evaluated with long-term follow-up.

Acknowledgments. We thank physical therapists and neonatologists at Aker, Ullevaal, and SiA hospitals for recruiting the infants and providing clinical information. We thank Vidar Jacobsen and his team at the Norwegian University of Sports and Physical Education, Oslo, for skillful technical assistance in the collection of kinematic data, Kristin Gillebo for participation in the Bayley assessments, Ingvil Øien for assistance in the clinical evaluations, and Lidy Kingma-Balkema for technical assistance in the preparation of the figure.

\section{REFERENCES}

1. Ornstein M, Ohlsson A, Edmonds J, Asztalos E 1991 Neonatal follow-up of very low birthweight/extremely low birthweight infants to school age: a critical overview. Acta Paediatr Scand 80:741-748

2. Herrgård E, Luoma L, Tuppurainen K, Karjalainen S, Martikainen A 1993 Neurodevelopmental profile at five years of children born at $\leq 32$ weeks gestation. Dev Med Child Neurol 35:1083-1096

3. Pinto-Martin JA, Whitaker AH, Feldman JF, Van Rossem R, Paneth N 1999 Relation of cranial ultrasound abnormalities in low-birthweight infants to motor or cognitive performance at ages 2, 6, and 9 years. Dev Med Child Neurol 41:826-833

4. de Groot L, Van der Hoek AM, Hopkins B, Touwen BCL 1992 Development of the relationship between active and passive muscle power in preterms after term age. Neuropediatrics 23:298-305

5. de Groot L, Van der Hoek AM, Hopkins B, Touwen BCL 1993 Development of muscle power in preterm infants: individual trajectories after term age. Neuropediatrics 24:68-74

6. Drillien CM 1972 Abnormal neurological signs in the first year of life in low birthweight infants: possible prognostic significance. Dev Med Child Neurol 14:575584

7. Sommerfelt K, Pedersen P, Ellertsen B, Markestad T 1996 Transient dystonia in non-handicapped low-birthweight infants and later neurodevelopment. Acta Paediatr 85:1445-1449

8. van der Fits I, Hadders-Algra M 1999 Development of postural adjustments during reaching in preterm infants. Pediatr Res 46:1-7

9. Plantinga Y, Perdock J, de Groot L 1997 Hand function in low-risk preterm infants: its relation to muscle power. Dev Med Child Neurol 39:6-11

10. Samsom JF, de Groot L 2000 The influence of postural control on motility and hand function in a group of "high risk" preterm infants at 1 year of age. Early Hum Dev 60:101-113

11. Gorga D, Stern FM, Ross G, Nagler W 1988 Neuromotor development of preterm and full-term infants. Early Hum Dev 18:137-149

12. Piper MC, Byrne PJ, Darrah, J Watt MJ 1989 Gross and fine motor development of preterm infants at eight and 12 months of age. Dev Med Child Neurol 31:591-597 
13. von Hofsten C 1979 Development of visually directed reaching: the approach phase. J Hum Mov Stud 5:160-168

14. von Hofsten C 1991 Structuring of early reaching movements: a longitudinally study. J Mot Behav 23:280-292

15. Fetters L, Todd J 1987 Quantitative assessment of infant reaching movements. J Mot Behav 19:147-166

16. Thelen E, Corbetta D, Kamm K, Spencer JP, Schneider K, Zericke RF 1993 The transition to reaching: mapping intention to intrinsic dynamics. Child Dev 64:1058-1098

17. Fallang B, Saugstad OD, Hadders-Algra M 2000 Goal directed reaching and postural control in supine position in healthy infants. Behav Brain Res 115:9-18

18. Out L, Savelsbergh GJP, van Soest AJ, Hopkins B 1997 Influence of mechanical factors on movement units in infant reaching. Hum Mov Sci 16:733-748

19. Bayley N 1993 Bayley Scales of Infant Development Manual, 2nd Ed. The Psychological Corporation, Harcourt Brace \& Company, San Antonio, TX pp 1-367

20. Touwen BCL 1979 Examination of the child with minor neurological dysfunction, 2nd Ed. Clin Dev Med 71 Spastics International Medical Publications William Heinemann Medical Books, London, pp 1-123

21. Gerner EM, Katz-Salamon M, Hesser U, Söderman E, Forssberg H 1997 Psychomotor development at 10 months as related to neonatal health status: the Stockholm Neonatal Project. Acta Paediatr Suppl 419:37-43

22. Smith L, Ulvund SE, Lindemann R 1994 Very low birth weight infants $(<1501 \mathrm{~g})$ at double risk. J Dev Behav Pediatr 15):7-13

23. Evans N 1993 Diagnosis of patent ductus arteriosus in the preterm newborn. Arch Dis Child 68:58-61

24. Committee for the classification of retinopathy of prematurity: international classification of retinopathy of prematurity 1984 Arch Ophthalmol 102:1130-1134

25. Levene MI, Fawer C-L, Lamont RF 1982 Risk factors in the development of intraventricular haemorrhage in the preterm neonate. Arch Dis Child 57:410-417

26. Jongmans M, Henderson S, De Vries L, Dubowitz L 1993 Duration of periventricula densities in preterm infants and neurological outcome at six years of age. Arch Dis Child 69:9-13
27. Touwen B 1980 The preterm infant in the extrauterine environment. Implications for neurology. Early Hum Dev 4:287-300

28. Tsuneishi S, Casaer P 2000 Effects of preterm extrauterine visual experience on the development of the human visual system: a flash VEP study. Dev Med Child Neurol 42:663-668

29. van Hof van Duin J, Heersema DJ, Groenendal F, Baerts W, Fetter WP 1992 Visual field and grating acuity development in low-risk preterm infants during the first 21/2 years after term. Behav Brain Res 49:115-122

30. Eilers RE, Oller DK, Levine S, Basinger D 1993 The role of prematurity and socioeconomic status in the onset of canonical babbling in infants. Infant Behav Dev 26:297-315

31. Hadders Algra M, Huisjes HJ, Touwen BCL 1988 Perinatal correlates of major and minor neurological dysfunction at school age: a multivariate analysis. Dev Med Child Neurol 30:472-481

32. Darrah J, Redfern L, Maguire TO, Beaulne AP, Watt J 1998 Intra-individual stability of rate of gross motor development in full-term infants. Early Hum Dev 52:169-179

33. van der Fits IBM, Otten E, Klip AWJ, van Eykern LA, Hadders-Algra M 1999 The development of postural adjustments during reaching in 6- to 18-month-old infants. Evidence for two transitions. Exp Brain Res 126:517-528

34. Hadders-Algra M, Brogren E, Katz-Salamon M, Forssberg H 1999 Periventricular leucomalacia and preterm birth have different detrimental effects on postural adjustments. Brain 122:727-740

35. Hüppi PT, Schuknecht B, Boesch C, Bossi E, Felblinger J, Fusch C, Herschkowitz N 1996 Structural and neurobehavioral delay in postnatal brain development of preterm infants. Pediatr Res 39:895-901

36. van der Knaap MS 2001 Magnetic resonance in childhood white-matter disorders. Dev Med Child Neurol 43:705-712

37. Kloosterman GJ 1970 On intrauterine growth. Int J Gynaecol Obstet 8:895-912 